\title{
Gastrectomía por cáncer gástrico: abordaje mínimamente invasivo
}

\author{
Gastrectomy for gastric cancer: Minimally invasive approach
}

\author{
Raúl E. Pinilla-Morales ${ }^{1} \mathbb{D}$, Wilmar Martín-Montero² ${ }^{(\mathbb{D}}$, Helena Facundo-Navia ${ }^{3}$, \\ María E. Manrique-Acevedo ${ }^{3}$, Oscar A. Guevara-Cruz¹ $\mathbb{D}$, David R. Herrera-Mora ${ }^{3} \mathbb{D}$, \\ Ana D. Bonilla-Castañeda ${ }^{3} \mathbb{D}$, Mario Rey-Ferro ${ }^{3} \mathbb{D}$, Mario Abadía-Díaz ${ }^{3} \mathbb{D}$, \\ Juliana Rendón-Hernández ${ }^{3} \mathbb{D}$, Ricardo Sánchez-Pedraza ${ }^{3} \mathbb{D}$, Ricardo Oliveros-Wilches ${ }^{3} \mathbb{D}$
}

1 Médico, especialista en Cirugía general y Cirugía gastrointestinal y endoscopia digestiva. Departamento de Cirugía Gastrointestinal y Endoscopia Digestiva, Instituto Nacional de Cancerología; profesor, Universidad Nacional de Colombia, Bogotá D.C., Colombia.

2 Médico, especialista en Cirugía general, residente de segunda especialidad en Cirugía gastrointestinal y endoscopia digestiva, Universidad Militar Nueva Granada, Instituto Nacional de Cancerología, Bogotá D.C., Colombia.

3 Médico, especialista en Cirugía general y Cirugía gastrointestinal y endoscopia digestiva. Departamento de Cirugía Gastrointestinal y Endoscopia Digestiva, Instituto Nacional de Cancerología, Bogotá D.C., Colombia.

\section{Resumen}

Introducción. El cáncer gástrico en nuestro país es una de las neoplasias más comunes y su diagnóstico generalmente se realiza en estadios avanzados. El objetivo de este estudio fue describir las características sociodemográficas y clínicas, la experiencia quirúrgica, y las complicaciones en los pacientes con cáncer gástrico.

Métodos. Se presenta una serie de casos en la que se revisaron las historias clínicas de pacientes con diagnóstico histológico de adenocarcinoma gástrico, a quienes se les practicó gastrectomía mínimamente invasiva en el Instituto Nacional de Cancerología de Bogotá D.C., Colombia, entre enero de 2012 y diciembre de 2018.

Resultados. Se realizó gastrectomía por laparoscopia convencional en 31 pacientes (75,6 \%) y por laparoscopia asistida por robot en 10 pacientes (24,4 \%). Los estadios clínicos fueron IA en 20 pacientes (48,7\%), IB en tres (7,3\%), IIA en nueve $(21,9 \%)$, IIB en cinco $(12,2 \%)$ y IIIA en cuatro pacientes $(9,7 \%)$. Se realizaron 24 gastrectomías totales (58,5\%) y 17 distales (41,4\%). No hubo muertes intraoperatorias ni posoperatorias a 30 días. La disección ganglionar predominante fue D2 en el 92,6\% (n=38) de los casos. Se presentaron complicaciones posoperatorias en el 17,1\% (n=7).

Discusión. La gastrectomía por cáncer gástrico realizada por vía laparoscópica convencional y la asistida por robot, parecen ser procedimientos seguros y factibles. La determinación de supervivencia libre de enfermedad y mortalidad asociada a cáncer será necesaria para establecer la seguridad oncológica de este tipo de procedimientos en nuestro medio.

Palabras clave: cáncer gástrico; gastrectomía; total; distal; laparoscopia; robótica; complicaciones; mortalidad.

Fecha de recibido: 15/10/2020 - Fecha de aceptación: 15/02/2021 - Fecha de publicación en línea: 05/04/2021 Correspondencia: Raúl E. Pinilla-Morales, Calle 1 \# 9-85, Instituto Nacional de Cancerología, Bogotá, D.C., Colombia.

Teléfono: +57 4320160 Extensión 5405. Correo electrónico: rpinillam@cancer.gov.co

Citar como: Pinilla-Morales RE, Martin-Montero W, Facundo-Navia H, Manrique-Acevedo ME, Guevara-Cruz OA, Herrera-Mora DR, Bonilla-Castañeda AD, et al. Gastrectomía por cáncer gástrico: abordaje mínimamente invasivo. Rev Colomb Cir. 2021;36:446-56. https://doi.org/10.30944/20117582.806

Este es un artículo de acceso abierto bajo una Licencia Creative Commons - BY-NC-ND https://creativecommons.org/licenses/by-ncnd/4.0/deed.es 


\begin{abstract}
Introduction. In our country, gastric cancer is one of the most common neoplasms and its diagnosis is generally made in advanced stages. The objective of this study was to describe the sociodemographic and clinical characteristics, surgical experience, and complications in patients with gastric cancer. In our country, gastric cancer is one of the most common neoplasms and its diagnosis is generally made in advanced stages. The objective of this study was to describe the sociodemographic and clinical characteristics, surgical experience, and complications in patients with gastric cancer.
\end{abstract}

Methods. A series of cases is presented in which the medical records of patients with a histological diagnosis of gastric adenocarcinoma, who underwent minimally invasive gastrectomy at the National Cancer Institute of Bogotá, Colombia, between January 2012 and December 2018.

Results. Conventional laparoscopic gastrectomy was performed in 31 patients (75.6\%) and by robot-assisted laparoscopy in 10 patients (24.4\%). The clinical stages were IA in 20 patients (48.7\%), IB in three (7.3\%), IIA in nine (21.9\%), IIB in five (12.2\%), and IIIA in four patients (9.7\%). Twenty-four total gastrectomies (58.5\%) and 17 distal gastrectomies (41.4\%) were performed. There were no intraoperative or postoperative deaths at 30 days. The predominant lymph node dissection was D2 in $92.6 \%(n=38)$ of the cases. Postoperative complications occurred in $17.1 \%(\mathrm{n}=7)$.

Discussion. Gastrectomy due to gastric cancer, performed by conventional laparoscopic and robot-assisted approaches, appear to be safe and feasible procedures. The determination of disease-free survival and cancerassociated mortality will be necessary to establish the oncological safety of this type of procedure in our environment.

Keywords: gastric cancer; gastrectomy; total; distant; laparoscopy; robotics; complications; mortality.

\section{Introducción}

La gastrectomía con vaciamiento ganglionar es el pilar fundamental del tratamiento curativo de los pacientes afectados por cáncer gástrico en estadios operables. Las técnicas mínimamente invasivas brindan resultados similares a los procedimientos abiertos desde el punto de vista oncológico, con menor sangrado, menor dolor y recuperación más rápida ${ }^{1,2}$. Kitano et al., reportaron la primera gastrectomía distal para el tratamiento de cáncer gástrico asistida por laparoscopia, realizada en $1991^{3}$, y Azagra et al., presentaron la primera serie por mínima invasión, que incluyó una gastrectomía total por cáncer gástrico ${ }^{4}$, y desde entonces se han realizado múltiples estudios que han logrado incluir los procedimientos mínimamente invasivos como una alternativa a la cirugía abierta, para el manejo de ésta enfermedad ${ }^{5}$.

Hashizume et al., ${ }^{6}$, realizaron la primera gastrectomía robótica en 2002, técnica que se ha consolidado como otra opción que puede superar limitaciones propias de los procedimientos laparoscópicos convencionales, como visión en dos dimensiones, amplitud y rango de movimientos reducidos y menor ergonomía, lo que se hace evidente particularmente durante momentos complejos, como la linfadenectomía y la reconstrucción ${ }^{7,8}$. Algunas de las características de las plataformas robóticas que pueden contribuir a optimizar el manejo de mínima invasión en pacientes afectados por cáncer gástrico, son la visión en tres dimensiones, la magnificación 10X, la disponibilidad de instrumentos con un mayor rango de movimiento a través de su rotación de $540^{\circ}$ y articulación de $180^{\circ} 9$.

La implementación segura de la laparoscopia en el manejo de estos pacientes es esencial para evitar comprometer los resultados oncológicos. La literatura disponible ha demostrado beneficios en lesiones tempranas y ya se obser- 
van resultados a corto plazo con supervivencia adecuada a tres años en lesiones avanzadas ${ }^{10,11}$. El porcentaje de pacientes diagnosticados en estadios tempranos en nuestro medio es bajo ${ }^{12}$, lo que ocasiona que con mayor frecuencia tengamos la necesidad de llevar a cirugía a pacientes con lesiones avanzadas.

En Suramérica existen reportes de procedimientos mínimamente invasivos para el manejo de cáncer gástrico ${ }^{13-15}$, pero no se conocen informes de resultados específicos para los procedimientos de mínima invasión realizados en nuestro país ${ }^{16}$.

\section{Métodos}

Estudio observacional descriptivo de tipo serie de casos, realizado entre enero de 2012 y diciembre de 2018. Los datos fueron tomados de las historias clínicas, revisando las características demográficas, clínicas y de patología quirúrgica de los pacientes con adenocarcinoma gástrico, llevados a gastrectomía por técnica mínimamente invasiva, con intención curativa, en el Instituto Nacional de Cancerología, de Bogotá, D.C., Colombia, los cuales fueron registrados en una base de datos en el sistema REDCap ${ }^{\circledR}$ diseñada para tal fin. El análisis estadístico se realizó con el programa versión STATA versión 14.

Por medio de tomografía computarizada toracoabdominal se determinó la clasificación TNM preoperatoria de acuerdo a la octava edición ${ }^{17}$, y se incluyeron estadios clínicos I, II, y III sin lesiones tumorales mayores a seis $\mathrm{cm}$ y sin compromiso extra gástrico. Las técnicas quirúrgicas empleadas fueron la laparoscopia convencional y la laparoscopia asistida por robot, ésta última iniciada en octubre de 2017, siendo la primera institución pública del país en poner en marcha un programa de cirugía robótica, siguiendo los lineamientos internacionales disponibles para el desarrollo de este procedimiento ${ }^{18}$. Los criterios para la utilización de la plataforma robótica o para realizar la laparoscopia convencional fueron los mismos. Los tipos de gastrectomía realizados fueron total y distal con vaciamiento ganglionar D2 según la ubicación del tumor.

\section{Técnicas quirúrgicas}

Procedimiento laparoscópico convencional. El procedimiento laparoscópico se realizó con base en la experiencia reportada por las instituciones asiáticas ${ }^{19}$. Inicialmente se realizó una incisión umbilical de dos cm y con técnica abierta se avanzó un trocar de $10 \mathrm{~mm}$ a la cavidad abdominal, con insuflación de $\mathrm{CO}_{2}$ hasta una presión de $14 \mathrm{mmHg}$. Luego de avanzar el lente óptico y bajo visión laparoscópica, se avanzó un trocar de 12 $\mathrm{mm}$ en la línea axilar anterior derecha y otro de $12 \mathrm{~mm}$ en la línea axilar anterior izquierda, un trocar de $5 \mathrm{~mm}$ en el flanco izquierdo y otro trocar de $5 \mathrm{~mm}$ en la región subxifoidea.

Para la omentectomía parcial, se comenzó la disección del epiplón mayor a cuatro $\mathrm{cm}$ de la arcada gastroepiploica. Para la omentectomía total, se realizó decolamiento coloepiploico con energía monopolar y bisturí ultrasónico, en dirección al polo inferior del bazo, se identificaron y se ligaron con Hem $\mathrm{O}$ lok ${ }^{\circledR}$ la raíz de los vasos gastroepiploicos izquierdos. La curvatura mayor se liberó hasta unos $5 \mathrm{~cm}$ del margen de resección en caso de gastrectomía distal o hasta el ángulo de His en caso de gastrectomía total, y se continuó la disección del ligamento gastrocólico en dirección pilórica, identificando y ligando los vasos gastroepiploicos derechos en su raíz, incluyendo la estación ganglionar No 6, disecando los tejidos sobre la vena pancreáticoduodenal anterior y superior. Luego se siguió la disección sobre la arteria gastroduodenal en la pared posterior del duodeno, para después realizar la sección del borde superior del ligamento hepatoduodenal, y la sección del duodeno un $\mathrm{cm}$ distal al píloro, con sutura mecánica lineal de $60 \mathrm{~mm}$.

Se inició la disección de la arteria hepática propia hasta identificar la raíz de la arteria gástrica derecha, la cual se seccionó previo control con clip plástico en su origen. Se realizó la disección de los tejidos laterales a la arteria hepática propia, liberando el aspecto lateral izquierdo de la vena porta, y obteniendo los ganglios de la estación No $12 \mathrm{a}$, después se expuso la vena gástrica izquierda hacia el margen superior de la arteria hepática común, en el borde superior del páncreas, durante la 
disección de los ganglios linfáticos alrededor de la arteria hepática común, traccionando el estómago en sentido anterior.

La arteria gástrica izquierda se seccionó en la raíz, luego de disecar el tejido linfático y el tejido graso en forma circunferencial, resecando los ganglios de la estación No 7, completando así el vaciamiento supra pancreático (figura 1). Se continuó la disección retroperitoneal hasta el pilar derecho y se liberó completamente la curva menor hasta la unión esofagogástrica. Se realizó sección del esófago distal o del cuerpo gástrico según la extensión de la resección, con sutura mecánica lineal laparoscópica de $60 \mathrm{~mm}$. La reconstrucción del tracto gastrointestinal en caso de anastomosis esófago-yeyunal, se realizó con dispositivo de sutura circular $\mathrm{OrVil}^{\mathrm{TM}} \mathrm{de} 25 \mathrm{~mm}$, previa introducción del yunque en el asa del yeyuno, con realización de anastomosis esófago-yeyunal termino lateral. Luego se cerró el bastón yeyunal con sutura mecánica lineal de $45 \mathrm{~mm}$. En caso de anastomosis gastro-yeyunal, se hizo enterotomía y gastrotomía con posterior introducción de sutura mecánica lineal de $60 \mathrm{~mm}$ y cierre del defecto con sutura absorbible 3-0.

Excepto en casos de procedimientos paliativos o en pacientes ancianos, se realizó reconstrucción en Y de Roux, a los $70 \mathrm{~cm}$ de esta anastomosis, se

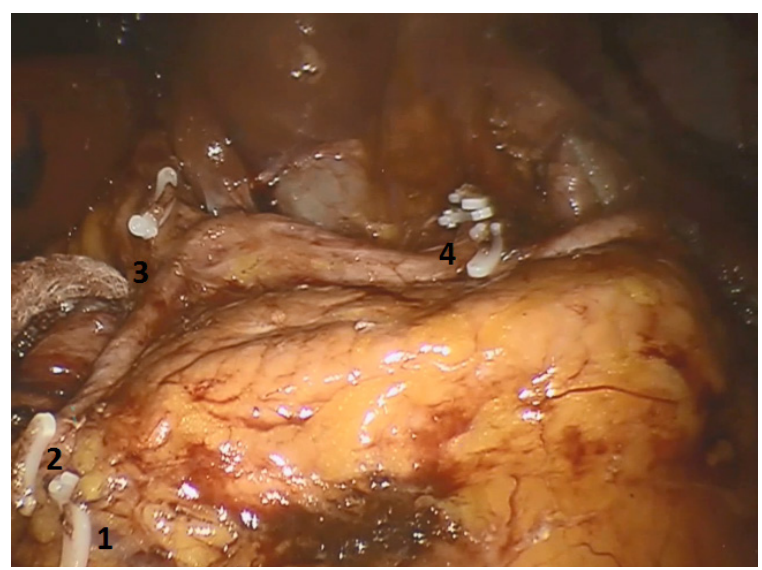

Figura 1. Vaciamiento suprapancreático finalizado. 1. Muñón de vena gastroepiplóica derecha. 2. Muñón de arteria gastroepiplóica derecha. 3. Muñón de arteria gástrica derecha. 4. Muñón de vasos gástricos izquierdos. realizó anastomosis yeyuno-yeyunal latero lateral con sutura lineal laparoscópica de $60 \mathrm{~mm}$ y cierre del defecto con PDS 3-0 intracorpóreo. Se amplió la incisión umbilical, se insertó un protector de herida mediano, y bajo guía laparoscópica se realizó extracción de la pieza quirúrgica, hemostasia, y colocación de drenajes en los procedimientos de gastrectomía total. La línea media se cerró con sutura absorbible y la piel con puntos no absorbibles.

\section{Procedimiento laparoscópico asistido por} robot. Se siguieron los lineamientos con respecto a la técnica, publicados previamente por el profesor Woo Jin Hyung et al., ${ }^{20}$. Se utilizó el sistema Da Vinci $\mathrm{Si}^{\circledR}$ (Sunnyvale, CA, EE. UU.), bajo anestesia general, en decúbito supino y con posición de Trendelemburg reverso de $15^{\circ}$ con "docking" cefálico, con la consola del cirujano ubicada a dos metros. Se utilizaron todos los brazos robóticos iniciando por técnica abierta con el trocar de 12 mm para la cámara infraumbilical, insuflación con $\mathrm{CO}_{2}$ hasta una presión de $14 \mathrm{mmHg}$. Bajo visión directa se introdujo un trocar de $12 \mathrm{~mm}$ para la segunda cámara y se utilizaron tres trocares de 8 $\mathrm{mm}$ y otro auxiliar de $12 \mathrm{~mm}$ (figura 2).

Se utilizaron pinzas robóticas tipo Maryland con energía bipolar, y bisturí ultrasónico para la disección, y cuando fue necesario, porta agujas para el cierre de las brechas, luego de las anasto-

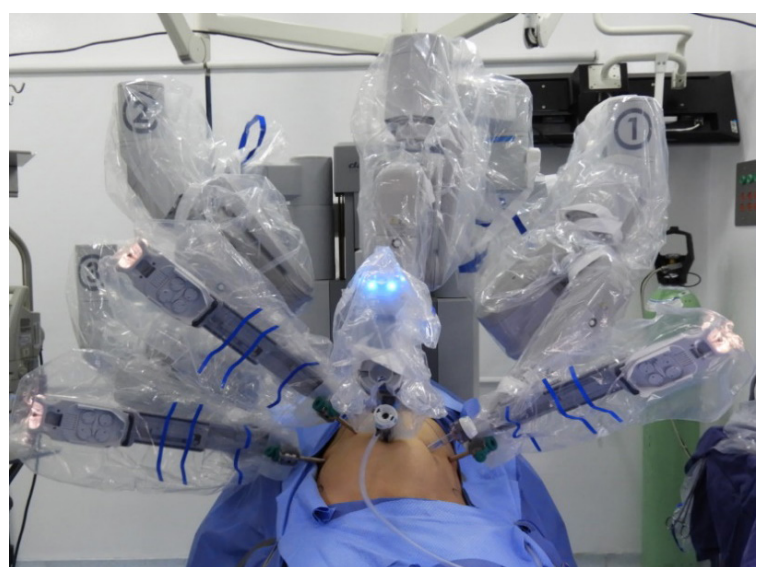

Figura 2. "Docking" con plataforma da $\mathrm{Vinci}^{\circledR} \mathrm{Si}$ en gastrectomía asistida por robot. 
mosis. El procedimiento se llevó a cabo siguiendo los mismos pasos de la técnica laparoscópica convencional y siguiendo los principios oncológicos de los procedimientos abiertos.

\section{Cuidados posoperatorios}

En el posoperatorio todos los pacientes requirieron manejo analgésico endovenoso. La vía oral con dieta líquida se inició en los pacientes con gastrectomía distal, una vez se documentó la presencia de tránsito intestinal. En los pacientes con gastrectomía total, se realizó al cuarto día un estudio de vías digestivas para verificar la integridad de la anastomosis. Las complicaciones fueron clasificadas con la escala de Clavien-Dindo ${ }^{21}$, se describieron complicaciones quirúrgicas, médicas y mortalidad durante el primer mes tras la cirugía. Se determinaron los resultados oncológicos iniciales para cada procedimiento con los bordes de sección y la disección linfática.

\section{Resultados}

Durante el período evaluado se identificaron 41 pacientes sometidos a gastrectomía, para tratamiento de adenocarcinoma gástrico. El 75,6 \% de los pacientes $(\mathrm{n}=31)$ fueron operados por laparoscopia convencional y el 24,4\% asistidos por robot $(\mathrm{n}=10)$. La mediana de edad fue similar entre los dos grupos según la técnica quirúrgica. El índice de masa corporal (IMC) también tuvo valores similares en ambos grupos (tabla1).

La localización más frecuente del tumor fue en la región proximal, con un 41,4 \% ( $\mathrm{n}=17)$, seguido por la región distal con un $39 \%$ (n=16). El $40 \%$ de los pacientes presentaban lesiones tempranas y en el mismo porcentaje no se encontró compromiso ganglionar en el estudio patológico (tabla 2).

En solo dos pacientes se cambió la intención del procedimiento a paliativo, uno con hallazgos intraoperatorios de linitis plástica, siembras peritoneales perigástricas y con sintomatología intensa, en quien además se documentó el borde de sección duodenal y esofágico positivos. El segundo por compromiso metastásico en el ligamento redondo, el cual se resecó en el mismo acto. Según la clasificación de Lauren, el tipo histológico más
Tabla 1. Características sociodemográficas y clínicas de los pacientes sometidos a gastrectomía por técnica de mínima invasión.

\begin{tabular}{lcc}
\hline \multicolumn{1}{c}{ Variable } & $\begin{array}{c}\text { Laparoscopia } \\
\text { convencional } \\
\mathbf{n = 3 1}(\%)\end{array}$ & $\begin{array}{c}\text { Asistida por } \\
\text { robot } \\
\mathbf{n = 1 0}(\%)\end{array}$ \\
\hline $\begin{array}{l}\text { Edad (años), } \\
\text { (mediana/ RIQ) }\end{array}$ & $12(56 \%)$ & $19(59 \%)$ \\
Género & & \\
Hombres & $15(48,4 \%)$ & $3(30 \%)$ \\
Mujeres & $16(51,6 \%)$ & $7(70 \%)$ \\
$\begin{array}{l}\text { IMC (kg/m2), } \\
\text { (mediana/ RIQ) }\end{array}$ & $5,3(23,4 \%)$ & $1,4(23,1 \%)$ \\
Comorbilidades & & \\
No & $19(61,3 \%)$ & $8(80 \%)$ \\
Si & $12(38,7 \%)$ & $2(20 \%)$ \\
\hline
\end{tabular}

frecuente fue el intestinal en un 65,8 \% ( $n=27)$. El compromiso linfovascular fue reportado en el $60,9 \%(n=25)$ y el perineural en el $39 \%(n=16)$.

En los procedimientos asistidos por robot, se realizó una mayor proporción de gastrectomías totales. En los laparoscópicos convencionales, el número entre gastrectomías totales y distales fue equivalente, la mediana de sangrado fue similar en ambos grupos. La reconstrucción en Y de Roux fue predominante en ambos grupos y con respecto a la extensión del vaciamiento solo en tres pacientes se realizó un vaciamiento inferior D1+. Se empleó un mayor tiempo quirúrgico en los procedimientos asistidos por robot $(\mathrm{p}<0,05)$. El número de ganglios resecados fue discretamente mayor en el grupo asistido por robot, con una mediana de 37 versus 32, diferencia no significativa estadísticamente. Se realizó omentectomía total en más del $80 \%$ de los casos ( $n=33$ ). La mediana de hospitalización fue de nueve días en los procedimientos laparoscópicos y de siete días en los asistidos por robot (tabla 3). No se presentó ninguna conversión ni muerte durante el procedimiento.

\section{Terapia complementaria}

Con respecto a la terapia médica asociada, solo un paciente con un tumor proximal recibió esquema neoadyuvante con quimio y radioterapia. Otro pa- 
ciente fue sometido a esquema perioperatorio con quimioterapia antes y después de la cirugía, cuya laparoscopia de estadificación inicial demostró una lesión con resecabilidad limítrofe. El 24,3 \% de los pacientes $(\mathrm{n}=10)$ se sometieron a adyuvancia, tres con quimioterapia y radioterapia y siete solo con quimioterapia.

\section{Complicaciones}

Se encontraron cuatro complicaciones grado I-II según la clasificación de Clavien-Dindo, todas ellas en el grupo de pacientes llevados a laparoscopia convencional, una complicación grado III-IV en el grupo de laparoscopia convencional y otra en el grupo asistido por robot, que correspondió a una fuga del muñón duodenal y requirió una nueva intervención por medio de laparotomía para realizar el drenaje de la colección. Solo se presentó una fuga de la anastomosis esófago-yeyunal en el grupo de laparoscopia convencional, la cual se resolvió de manera conservadora. No ocurrió mortalidad intraoperatoria ni durante los 30 días posteriores a la cirugía.

Tabla 2. Clasificación pTNM de los pacientes sometidos a gastrectomía por técnica de mínima invasión.

\begin{tabular}{lcc}
\hline & $\begin{array}{c}\text { Laparoscopia convencional } \\
\mathbf{n = 3 1}(\%)\end{array}$ & $\begin{array}{c}\text { Asistida por robot } \\
\mathbf{n}=10(\%)\end{array}$ \\
\hline Estadio Tumoral (pT) & & \\
pT0 & $2(6,5 \%)$ & 0 \\
pTis & $2(6,5 \%)$ & 0 \\
pT1a & $5(16,1 \%)$ & $2(20 \%)$ \\
pT1b & 0 & $2(20 \%)$ \\
pT2 & $7(22,6 \%)$ & 0 \\
pT3 & $10(32,3 \%)$ & $1(10 \%)$ \\
pT4a & $5(16,1 \%)$ & $4(40 \%)$ \\
pT4b & 0 & $1(10 \%)$ \\
Estadio ganglionar (pN) & & \\
pN0 & $13(41,9 \%)$ & $4(40 \%)$ \\
pN1 & $4(12,9 \%)$ & $1(10 \%)$ \\
pN2 & $9(29 \%)$ & $1(10 \%)$ \\
pN3a & $4(12,9 \%)$ & $2(20 \%)$ \\
pN3b & $1(3,2 \%)$ & $2(20 \%)$ \\
Estadio clínico & & $1(10 \%)$ \\
0 & 0 & 0 \\
IA & $7(22,6 \%)$ & $4(40 \%)$ \\
IB & $6(19,4 \%)$ & 0 \\
IIA & $2(6,5 \%)$ & 0 \\
IIB & $2(6,5 \%)$ & $2(20 \%)$ \\
IIIA & $10(32,3 \%)$ & $1(10 \%)$ \\
IIIB & $2(6,5 \%)$ & $1(10 \%)$ \\
IIIC & $1(3,2 \%)$ & $1(10 \%)$ \\
IV & $1(3,2 \%)$ & \\
\hline
\end{tabular}


Tabla 3. Resultados quirúrgicos de los pacientes sometidos a gastrectomía por técnica de mínima invasión.

\begin{tabular}{lcc}
\hline & $\begin{array}{c}\text { Laparoscopia convencional } \\
\mathbf{n = 3 1}(\%)\end{array}$ & $\begin{array}{c}\text { Asistida por Robot } \\
\mathbf{n = 1 0}(\%)\end{array}$ \\
\hline Tipo de resección & $16(51,6 \%)$ & $7(70 \%)$ \\
$\quad$ Gastrectomía Total & $15(48,4 \%)$ & $3(30 \%)$ \\
Gastrectomía Distal & $200(200)$ & $200(150)$ \\
Sangrado (cc) mediana (RIQ) & $340(90)$ & $452,5(85)$ \\
Tiempo operatorio (minutos) mediana (RIQ) & & $10(100 \%)$ \\
Linfadenectomía & $28(90,3 \%)$ & 0 \\
D2 & $3(9,7 \%)$ & $37(15)$ \\
$\quad$ D1+ & $32(21)$ & $3(30 \%)$ \\
Número de ganglios resecados mediana (RIQ) & & $7(70 \%)$ \\
Omentectomía & $5(16,1 \%)$ & $7(3)$ \\
Parcial & $26(83,9 \%)$ & \\
Completa & $9(4)$ & \\
Tiempo de hospitalización \\
(días), mediana (RIQ)
\end{tabular}

*Suma de rangos con signo, $Z=3,34 ; p=0,0004$

\section{Discusión}

Los resultados del presente estudio sugieren que el método laparoscópico convencional y el asistido por robot, para el manejo del cáncer gástrico mediante gastrectomía, son métodos seguros. La selección de los pacientes incluyó lesiones no abultadas y sin compromiso ganglionar extenso, con intención preoperatoria de manejo curativo.

En esta serie se observó un 17,1\% de complicaciones $(\mathrm{n}=7)$, valor no distante de lo reportado previamente por el grupo coreano de estudio de cirugía gastrointestinal laparo-endoscópico (Korean Laparoendoscopic Gastrointestinal Surgery Study Group [KLASS]) quienes informaron una morbilidad global del $13 \%$ en lesiones tempranas y de $15,7 \%$ en lesiones avanzadas ${ }^{22,23}$. El grupo CLASS (The Chinese Laparoscopic Gastrointestinal Surgery Study) también reportó una morbilidad en el grupo laparoscópico del 15,2 \% ${ }^{24}$.

En relación a las complicaciones Grado IIIIV de la clasificación de Clavien-Dindo, hemos encontrado un 7,3\% ( $n=3)$, valor superior al informado por el grupo Japonés en el JCOG0912 que solo incluyó gastrectomías distales, y quie- nes informaron un $2 \%$ de complicaciones ${ }^{25}$. No ocurrió mortalidad intraoperatoria ni durante los primeros 30 días, cifras similares a las publicadas por Cheng et al., en un meta-análisis con 3496 procedimientos laparoscópicos, con un rango de 0 a $3,3 \%{ }^{26}$.

Una de las preocupaciones de los procedimientos mínimamente invasivos, específicamente con respecto a la gastrectomía total, es la fuga de la anastomosis esófago-yeyunal. En este grupo de pacientes se encontró solo un caso, configurando una incidencia del $0,04 \%$, valor por debajo del $18,8 \%$ reportado por Hyung et al., en el KLASS- $03^{27}$; el manejo se dio de forma conservadora, prolongando el tiempo de nutrición enteral $y$ verificando la ausencia de fuga en el control por imágenes.

En quienes la intención del tratamiento fue curativo, y siguiendo las recomendaciones de las distintas guías para el manejo del cáncer gástrico, se obtuvo siempre al menos 16 ganglios, siendo clasificada como D2, según las estaciones ganglionares disecadas, con márgenes de resección negativos en todos los $\operatorname{casos}^{28-31}$. 
Los resultados oncológicos de esta serie a muy corto plazo, sugieren seguridad y factibilidad de la cirugía mínimamente invasiva, comparada con procedimientos abiertos, según lo encontrado en distintos ensayos clínicos publicados por grupos asiáticos ${ }^{10,22,25}$.

Es de suma importancia reconocer que, en la actualidad, no se considera aún este tipo de procedimientos como alternativa del tratamiento de las lesiones avanzadas. Estos procedimientos deberían realizarse solamente en centros de referencia, con un volumen adecuado, no solo para la institución, sino para sus cirujanos, y con la cuidadosa selección de los pacientes, incluyéndolos en bases de datos, para realizar un seguimiento estricto de la evolución y de los resultados ${ }^{32}$. Es importante además, informar a los pacientes que aún no existen estudios que identifiquen ventajas, ni desde el punto de vista oncológico ni reconstructivo, al ofrecerles éste tipo de intervenciones ${ }^{33}$.

La ausencia de conversión a cirugía abierta en este trabajo puede estar explicada por la exclusión de pacientes con lesiones abultadas y con gran carga ganglionar, así como la experiencia previa con el manejo abierto de éstos procedimientos y la utilización frecuente de técnicas mínimamente invasivas en otros procedimientos oncológicos digestivos ${ }^{34-38}$. El tiempo operatorio prolongado en ambos tipos de procedimientos (especialmente en los robóticos), con respecto a los procedimientos abiertos, es una de las desventajas de estas técnicas ${ }^{39,40}$.

A pesar de los resultados adecuados, se pueden mencionar varias limitaciones como el número pequeño de casos, el carácter retrospectivo del análisis y la falta de comparación con procedimientos abiertos. Por tratarse de una experiencia inicial, y dada la necesidad de pacientes con patología menos compleja, no se evaluaron los criterios de selección para que los pacientes fueran llevados a procedimiento laparoscópico convencional o asistido por robot, lo que pudo ocasionar sesgo de selección. El seguimiento de los pacientes, así como el análisis de la terapia complementaria recibida, se encargarán de definir la seguridad oncológica a mediano y largo plazo, con la identificación de tiempo libre de recurrencia y de supervivencia específica para cáncer.

\section{Conclusiones}

La gastrectomía mínimamente invasiva como parte del tratamiento del adenocarcinoma gástrico temprano y avanzado mostró ser segura y factible. Los resultados de patología quirúrgica confirmaron recuentos ganglionares adecuados, con márgenes de resección negativos cuando se ofreció a pacientes con intención curativa.

Los resultados oncológicos precoces encontrados en los pacientes a quienes se les practicó cirugía robótica son similares con respecto a la cirugía laparoscópica convencional. El seguimiento juicioso de los pacientes, la determinación de la supervivencia libre de enfermedad y de la mortalidad asociada a cáncer, será la forma adecuada para demostrar la seguridad oncológica a mediano y largo plazo de estos procedimientos.

Esta es la primera serie informada específicamente para el tratamiento del cáncer gástrico por laparoscopia convencional y asistida por robot en el país.

\section{Agradecimientos}

A todo el personal administrativo y asistencial de salas de cirugía, y de la Unidad de cirugía gastrointestinal, gastroenterología y endoscopia digestiva del Instituto Nacional de Cancerología.

\section{Cumplimiento de normas éticas}

Consentimiento informado: Se diligenció el formato de consentimiento informado en todos los pacientes operados. Este trabajo se desarrolló contando con la aprobación del Comité de Ética del Instituto Nacional de Cancerología de Bogotá D.C.

Conflicto de interés: Los autores manifiestan que no tienen conflictos de interés de ninguna naturaleza para el desarrollo de la investigación.

Fuentes de financiación: Los recursos de financiación del proyecto provienen en su totalidad del Instituto Nacional de Cancerología. 


\section{Contribución de los autores:}

Concepción y diseño del estudio: Raúl E. Pinilla, Wilmar Martin Montero, Helena Facundo Navia, María E. Manrique-Acevedo, Oscar A. Guevara Cruz, David R. Herrera-Mora, Ana D. Bonilla-Castañeda, Mario Rey-Ferro, Mario Abadía-Díaz, Juliana Rendón-Hernández, Ricardo Sánchez Pedraza, Ricardo Oliveros-Wilches.

Adquisición de datos: Raúl E. Pinilla, Wilmar Martin Montero, Helena Facundo Navia, María E. ManriqueAcevedo, Oscar A. Guevara Cruz, David R. HerreraMora, Ana D. Bonilla-Castañeda, Mario Rey-Ferro, Mario Abadía-Díaz, Juliana Rendón-Hernández, Ricardo Sánchez Pedraza, Ricardo Oliveros-Wilches.

Análisis e interpretación de datos: Raúl E. Pinilla, Wilmar Martin Montero, Helena Facundo Navia, María E. Manrique-Acevedo, Oscar A. Guevara Cruz, David R. Herrera-Mora, Ana D. Bonilla-Castañeda, Mario Rey-Ferro, Mario Abadía-Díaz, Juliana Rendón-Hernández, Ricardo Sánchez Pedraza, Ricardo Oliveros-Wilches.

Redacción del manuscrito: Raúl E. Pinilla, Wilmar Martin Montero, Helena Facundo Navia, María E. ManriqueAcevedo, Oscar A. Guevara Cruz, David R. HerreraMora, Ana D. Bonilla-Castañeda, Mario Rey-Ferro, Mario Abadía-Díaz, Juliana Rendón-Hernández, Ricardo Sánchez Pedraza, Ricardo Oliveros-Wilches.

Revisión crítica: Raúl E. Pinilla, Wilmar Martin Montero, Helena Facundo Navia, María E. Manrique-Acevedo, Oscar A. Guevara Cruz, David R. Herrera-Mora, Ana D. BonillaCastañeda, Mario Rey-Ferro, Mario Abadía-Díaz, Juliana Rendón-Hernández, Ricardo Sánchez Pedraza, Ricardo Oliveros-Wilches.

\section{Referencias}

1. Lu W, Gao J, Yang J, Zhang Y, Lv W, Mu J, et al. Longterm clinical outcomes of laparoscopy-assisted distal gastrectomy versus open distal gastrectomy for early gastric cancer. Medicine. 2016;95:e3986. https://doi.org/10.1097/MD.0000000000003986

2. Beyer K, Baukloh AK, Kamphues C, Seeliger H, Heidecke $\mathrm{CD}$, Kreis ME, et al. Laparoscopic versus open gastrectomy for locally advanced gastric cancer: A systematic review and meta-analysis of randomized controlled studies. World J Surg Oncol. 2019;17:1-19. https://doi.org/10.1186/s12957-019-1600-1

3. Kitano S, Iso Y, Moriyama M, Sugimachi K. Laparoscopy-assisted Billroth I gastrectomy. Surg Laparosc Endosc. 1994;4:146-8.

4. Azagra JS, Goergen M, De Simone P, Ibañez-Aguirre J. Minimally invasive surgery for gastric cancer.
Surg Endosc. 1999;13:351-7. https://doi.org/10.1007/s004649900988

5. Hosono S, Arimoto Y, Ohtani H, Kanamiya Y. Meta-analysis of short-term outcomes after laparoscopy-assisted distal gastrectomy. World J Gastroenterol. 2006;12:7676-83.

https://doi.org/10.3748/wjg.v12.i47.7676

6. Hashizume M, Shimada M, Tomikawa M, Ikeda Y, Takahashi I, Abe R, et al. Early experiences of endoscopic procedures in general surgery assisted by a computer-enhanced surgical system. Surg Endosc Other Interv Tech. 2002;16:1187-91.

https://doi.org/10.1007/s004640080154

7. Barchi LC, Jacob CE, José C, Bresciani C, Yagi OK, Mucerino DR, et al. Minimally invasive surgery for gastric cancer: time to change the paradigm. 2016;29:117-20. https://doi.org/10.1590/0102-6720201600020013

8. Wee IJY, Kuo LJ, Ngu JCY. A systematic review of the true benefit of robotic surgery: Ergonomics. Int J Med Robot Comput Assist Surg. 2020;16:1-10. https://doi.org/10.1002/rcs.2113

9. Liao G, Zhao Z, Khan M, Yuan Y, Li X. Comparative analysis of robotic gastrectomy and laparoscopic gastrectomy for gastric cancer in terms of their long-term oncological outcomes: A meta-analysis of 3410 gastric cancer patients. World J Surg Oncol. 2019;17:1-9 https://doi.org/10.1186/s12957-019-1628-2

10. Yu J, Huang C, Sun Y, Su X, Cao H, Hu J, et al. Effect of laparoscopic vs open distal gastrectomy on 3-year disease-free survival in patients with locally advanced gastric cancer: the CLASS-01 randomized clinical trial. JAMA - J Am Med Assoc. 2019;321:1983-92. https://doi.org/10.1001/jama.2019.5359

11. Hyung WJ, Yang H-K, Park Y-K, Lee H-J, An JY, Kim W, et al. Long-term outcomes of laparoscopic distal gastrectomy for locally advanced gastric cancer: the KLASS-02-RCT randomized clinical trial. J Clin Oncol. 2020;38:330413. https://doi.org/10.1200/JC0.20.01210

12. Oliveros R, Navarrera LF. Diagnóstico, estadificación y tratamiento del cáncer gástrico en Colombia desde 2004 a 2008 (REGATE - Colombia). Rev Colomb Gastroenterol. 2012;27:269-74.

13. Berrospi F, Celis J, Ruíz E, Payet E, Chávez I, Young F. Gastrectomía distal laparoscópica por cáncer gástrico. Experiencia inicial Rev Gastroenterol Peru. 2008;28:119-24.

14. Escalona PA, Báez VS, Pimentel MF, Calvo BA, Boza WC, Viñuela FE, et al. Gastrectomía laparoscópica en cáncer gástrico Rev Chil Cir. 2008;60:188-93. https://doi.org/10.4067/S0718-40262008000300004

15. Norero E, Báez S, Briceño E, Martínez C, Ceroni M, Escalona A, et al. Gastrectomía totalmente laparoscópica por neoplasias gástricas. Experiencia en un centro público. Rev Med Chile. 2015;143:281-8. https://doi.org/10.4067/S0034-98872015000300001 
16. Campillo J, Suárez L, Guevara R, Burgos R, Villegas C, Cendales M, et al. Supervivencia y complicaciones en pacientes con cáncer gástrico y de la unión gastroesofágica tratados con quimioterapia perioperatoria más cirugía comparada con cirugía más terapia adyuvante: estudio multicéntrico, Bogotá D.C., 2010-2017. Rev Colomb Cirugía. 2018;33:353-61. https://doi.org/10.30944/20117582.82

17. American College of Surgeons. AJCC cancer staging manual. Amin M, Edge S, Greene F, Byrd DR, Brookland RK, Washington MK, eds. 8th edition. Berlin: Springer; 2017. p. 203-220.

18. Advancing A, Invasive M, Worldwide G. Guidelines for privileging for robotic-assisted gynecologic laparoscopy AAGL advancing minimally invasive gynecology worldwide. J Minim Invasive Gynecol. 2014;21:157-67. https://doi.org/10.1016/j.jmig.2014.01.024

19. Son T, Kwon IG, Hyung WJ. Minimally invasive surgery for gastric cancer treatment: Current status and future perspectives. Gut Liver. 2014;8:229-36. https://doi.org/10.5009/gnl.2014.8.3.229

20. Hyung WJ, Woo Y, Noh SH. Robotic surgery for gastric cancer: A technical review. J Robot Surg. 2011;5:241-9. https://doi.org/10.1007/s11701-011-0263-x

21. Dindo D, Demartines N, Clavien P. Classification of Surgical Complications.2004;240:205-13. https://doi.org/10.1097/01.sla.0000133083.54934.ae

22. Kim HH, Han SU, Kim MC, Kim W, Lee HJ, Ryu SW, et al. Effect of laparoscopic distal gastrectomy vs open distal gastrectomy on long-term survival among patients with stage in gastric cancer: the KLASS-01 randomized clinical trial. JAMA Oncol. 2019;5:506-13. https://doi.org/10.1001/jamaoncol.2018.6727

23. Kurokawa Y, Katai H, Fukuda H, Sasako M. Phase II study of laparoscopy-assisted distal gastrectomy with nodal dissection for clinical stage I gastric cancer: Japan clinical oncology group study JCOG0703. Jpn J Clin Oncol. 2008;38:501-3. https://doi.org/10.1093/jjco/hyn055

24. Hu Y, Huang C, Sun Y, Su X, Cao H, Hu J, et al. morbidity and mortality of laparoscopic versus open D2 distal gastrectomy for advanced gastric cancer: a randomized controlled trial. J Clin Oncol. 2016;34:1350-7. https://doi.org/10.1200/JC0.2015.63.7215

25. Katai PH, Me JM, Katayama H, Morita S, Yamada T, Bando E, et al. Articles survival outcomes after laparoscopy-assisted distal gastrectomy versus open distal gastrectomy with nodal dissection for clinical stage IA or IB gastric cancer randomized controlled trial. Lancet Gastroenterol Hepatol 2019;1253:1-10. https://doi.org/10.1016/S2468-1253(19)30332-2

26. Cheng Q, Pang TCY, Hollands MJ, Richardson AJ, Pleass H, Johnston ES, et al. Systematic review and meta-analysis of laparoscopic versus open distal gastrectomy. J Gastrointest Surg. 2014;18:1087-99. https://doi.org/10.1007/s11605-014-2519-z

27. Yang HK, Hyung WJ, Han SU, Lee YJ, Park JM, Cho GS, et al. Comparison of surgical outcomes among different methods of esophagojejunostomy in laparoscopic total gastrectomy for clinical stage I proximal gastric cancer: results of a single-arm multicenter phase II clinical trial in Korea, KLASS 03. Surg Endosc. 2020;(0123456789). https://doi.org/10.1007/s00464-020-07480-0

28. Smyth EC, Verheij M, Allum W, Cunningham D, Cervantes A, Arnold D, et al. Gastric cancer: ESMO clinical practice guidelines for diagnosis, treatment and follow-up. Ann Oncol. 2016;27:v38-49.

https://doi.org/10.1093/annonc/mdw350

29. National Comprehensive Cancer Network. Gastric cancer. Version 3. Fecha de consulta: 8 de septiembre de 2020. Disponible en: https://jnccn.org/view/journals/ jnccn/14/10/article-p1286.xml.

30. Wang FH, Shen L, Li J, Zhou ZW, Liang H, Zhang XT, et al. The Chinese Society of Clinical Oncology (CSCO): clinical guidelines for the diagnosis and treatment of gastric cancer. Cancer Commun. 2019;39:10. https://doi.org/10.1186/s40880-019-0349-9

31. Japanese Gastric Cancer Association. Japanese gastric cancer treatment guidelines 2018 (5th edition). Gastric Cancer. 2021;24:1-21. https://doi.org/10.1007/s10120-020-01042-y

32. Berlth F, Grimminger PP. Comment Minimally invasive gastrectomy: time to change practice? Lancet Gastroenterol Hepatol. 2020;1253:9-10. https://doi.org/10.1016/S2468-1253(19)30361-9

33. Sasako M. Is there role for laparoscopic gastrectomy for advanced gastric cancer. EurJSurg Oncol.2017;43:965-7. https://doi.org/10.1016/j.ejso.2017.02.016

34. Obama K, Kim YM, Kang DR, Son T, Kim HI, Noh SH, et al. Long-term oncologic outcomes of robotic gastrectomy for gastric cancer compared with laparoscopic gastrectomy. Gastric Cancer. 2018;21:285-95. https://doi.org/10.1007/s10120-017-0740-7

35. Song J, Kang WH, Oh SJ, Hyung WJ, Choi SH, Noh SH. Role of robotic gastrectomy using da Vinci system compared with laparoscopic gastrectomy: initial experience of 20 consecutive cases. Surg Endosc. 2009;23:1204-11. https://doi.org/10.1007/s00464-009-0351-4

36. Park SS, Kim MC, Park MS, Hyung WJ. Rapid adaptation of robotic gastrectomy for gastric cancer by experienced laparoscopic surgeons. Surg Endosc. 2012;26:60-7. https://doi.org/10.1007/s00464-011-1828-5

37. Kim MS, Kim WJ, Hyung WJ, Kim HI, Han SU, Kim YW, et al. Comprehensive Learning Curve of Robotic Surgery: Discovery From a Multicenter Prospective Trial of Robotic Gastrectomy. Ann Surg. 2019 Sep 9. Volume 
publish ahead of print.

https://doi.org/10.1097/SLA.0000000000003583

38. Borráez Segura BA, Oliveros R, Sánchez R, Cajiao JC, Pinilla RE. First experience of minimally invasive surgery in the upper digestive tract. Rev Gastroenterol Peru. 2018;38:340-4.

https://doi.org/10.1097/SLA.0000000000003583

39. Shin HJ, Son SY, Wang B, Roh CK, Hur H, Han SU. Longterm comparison of robotic and laparoscopic gastrec- tomy for gastric cancer: a propensity score-weighted analysis of 2084 consecutive patients. Ann Surg. 2020 Mar 13.

https://doi.org/10.1097/SLA.0000000000003845

40. Qiu H, Ai JH, Shi J, Shan RF, Yu DJ. Effectiveness and safety of robotic versus traditional laparoscopic gastrectomy for gastric cancer: An updated systematic review and meta-analysis. J Cancer Res Ther. 2019;15:1450-63. https://doi.org/10.4103/jcrt.JCRT_798_18 